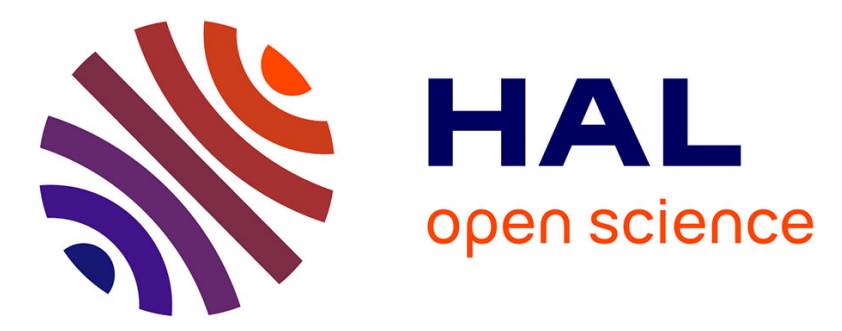

\title{
A Reconfigurable Cable-Driven Parallel Robot for Sandblasting and Painting of Large Structures
}

Lorenzo Gagliardini, Stéphane Caro, Marc Gouttefarde, Philippe Wenger, Alexis Girin

\section{- To cite this version:}

Lorenzo Gagliardini, Stéphane Caro, Marc Gouttefarde, Philippe Wenger, Alexis Girin. A Reconfigurable Cable-Driven Parallel Robot for Sandblasting and Painting of Large Structures. CableCon: Cable-Driven Parallel Robots, Aug 2014, Duisburg, Germany. pp.275-291, 10.1007/978-3-319-094892_20. lirmm-01221407

\section{HAL Id: lirmm-01221407 https://hal-lirmm.ccsd.cnrs.fr/lirmm-01221407}

Submitted on 10 Dec 2018

HAL is a multi-disciplinary open access archive for the deposit and dissemination of scientific research documents, whether they are published or not. The documents may come from teaching and research institutions in France or abroad, or from public or private research centers.
L'archive ouverte pluridisciplinaire HAL, est destinée au dépôt et à la diffusion de documents scientifiques de niveau recherche, publiés ou non, émanant des établissements d'enseignement et de recherche français ou étrangers, des laboratoires publics ou privés. 


\title{
A Reconfigurable Cable-Driven Parallel Robot for Sandblasting and Painting of Large Structures
}

Lorenzo Gagliardini, Stéphane Caro, Marc Gouttefarde, Philippe Wenger and Alexis Girin

\begin{abstract}
The research work presented in this paper introduces a Reconfigurable Cable Driven Parallel Robot (RCDPR) to be employed in industrial operations on large structures. Compared to classic Cable-Driven Parallel Robots (CDPR), which have a fixed architecture, RCDPR can modify their geometric parameters to adapt their own characteristics. In this paper, a RCDPR is intended to paint and sandblast a large tubular structure. To reconfigure the CDPR from one side of the structure to another one, one or several cables are disconnected from their current anchor points and moved to new ones. This procedure is repeated until all the sides of the structure are sandblasted and painted. The analysed design procedure aims at defining the positions of the minimum number of anchor points required to complete the task at hand. The robot size is minimized as well.
\end{abstract}

\section{Introduction}

Over the last decades, several companies faced the necessity to manufacture novel large industrial structures. Surface finishing, e.g. painting and sandblasting, can be part of the manufacturing process of those structures. According to the different structures, painting and sandblasting are usually performed by human operators,

Lorenzo Gagliardini, Alexis Girin

IRT Jules Verne, Chemin du Chaffault, 44340, Bouguenais, France e-mail: \{lorenzo.gagliardini, alexis.girin\}@ irt-jules-verne.fr

Stéphane Caro, Philippe Wenger

CNRS-IRCCyN, 1, rue de la Noë, 44321, Nantes Cedex 03, France e-mail: \{Stephane.Caro, Philippe.Wenger\}@irccyn.ec-nantes.fr

Marc Gouttefarde

Laboratoire d'Informatique, de Robotique et de Micro-électronique de Montpellier (LIRMM-CNRS-UM2), 161, rue Ada, 34392, Montpellier Cedex 05, France e-mail: marc.gouttefarde@lirmm.fr 
Fig. 1 Case study model. The structure is $20 \mathrm{~m}$ long, with a cross section of $10 \mathrm{~m} \times 10 \mathrm{~m}$.

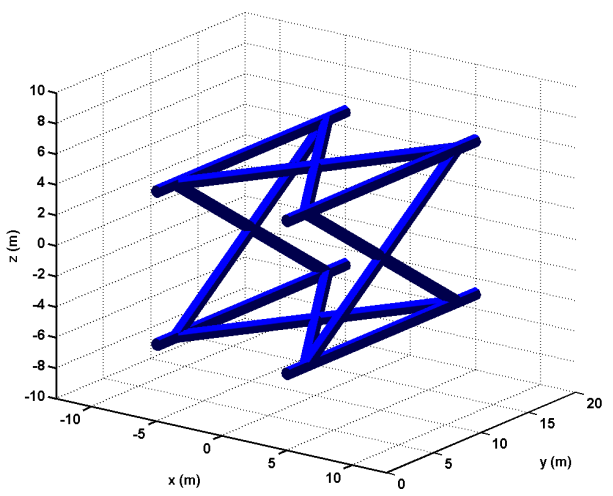

with the support of non-automatic machines. Alternative methods can improve the efficiency of these operations and release human operators from their unpleasant tasks. Cable Driven Parallel Robots (CDPR) are one of the possible solutions. By definition, CDPRs are parallel robots composed of a platform connected to a fixed base by cables (in this paper, the connection points between the cables and the base will be referred as anchor points). Sandblasting and painting tools can be embarked on the CDPR platform, which will follow the profile of the structure to be painted according to an off-line planned strategy.

Advantages of CDPRs are their wide workspace, the possibility to carry heavy loads and the simplicity of their mechanical components [7]. However, a limitation of CDPRs is the possible interferences between cables as well as between cables and the surrounding environment. Furthermore, the non-rigid nature of CDPR links requires a rigorous study of the force transmission characteristics.

The potentialities of CDPRs have already been proved in different industrial contexts [2], [10]. Other research studies are being performed in the framework of the European project CableBot [5]. Most of the previous works are dedicated to CDPRs with a fixed architecture and a fixed geometry (cable layout). This type of robots cannot always guarantee good performances when installed in cluttered environments. In this context, Reconfigurable Cable-Driven Parallel Robots (RCDPR) should represent a better solution. Indeed, they can modify their geometric parameters in order to adapt their characteristics or to avoid cable collisions.

One of the first works related to CDPR reconfigurability was part of the NIST RoboCrane project [3]. Further studies on reconfigurability have been performed by Zhuo et al. [17], as well as by Izard et al. [12] and Rosati et al. [16]. Rosati suggested to add additional DoF to a classical CDPR (e.g., moving the cable anchor points on a rail) and optimize analytically the robot properties, such a the payload capability. This method has been proved to be efficient for planar robots. However, it cannot be easily applied to three-dimensional case studies, where the analytic solution of the problem is very difficult to define. 
The research work presented in this paper focuses on the design of a RCDPR for sandblasting and painting of a three-dimensional tubular structure represented in Fig. 1. These operations are performed by appropriate tools embarked on the robot platform. The robot platform approaches each external side of the structure and the tools perform their work. Due to the structure complexity, reconfigurability is required in order to avoid cable collisions. Each external side of the structure is sandblasted and painted through a different configuration of the cable anchor points. To reconfigure the CDPR from one side of the structure to another one, one or several cables are disconnected from their current anchor points and moved to new ones. This procedure is repeated until all the sides of the structure are sandblasted and painted. The variables of the corresponding design problem are thus the Cartesian coordinates of the anchor points of the three required configurations associated to the paths $\mathscr{P}_{1}, \mathscr{P}_{2}$ and $\mathscr{P}_{3}$ illustrated in Fig. 2 .

In the present work, we aim at minimizing the total number of anchor points on the base, selecting the anchor point locations that can be shared between two or more configurations. Thereby, during a configuration change, not all the cable anchor points need to be modified. Furthermore, we also aim at minimizing the robot overall size. The feasibility of each configuration has to be guaranteed: cable interferences as well as cable collisions with the structure are not permitted. Moreover, a minimum platform pose precision is required.

This paper is organized as follows. Section 2 briefly introduces the industrial context and the problem at hand. Section 3 presents the RCDPR geometric, static and elastostatic models used in this paper. Section 4 provides a description of the selected design strategy. Section 5 presents the achieved results. Section 6 concludes this article.

\section{Context and Problem Description}

The structure selected for the given case study is $20 \mathrm{~m}$ long, with a cross section of $10 \mathrm{~m} \times 10 \mathrm{~m}$. The number of tubes to be painted is equal to twenty. Their diameter, $\phi_{s}$, is equal to $0.8 \mathrm{~m}$. The sandblasting and painting operations are realised indoor. The structure lies horizontally in order to reduce the dimensions of the painting workshop. The whole system can be described with respect to a fixed reference frame, $\mathscr{F}_{b}$, of origin $O_{b}$ and axes $\mathbf{x}_{b}, \mathbf{y}_{b}, \mathbf{z}_{b}$, as illustrated in Fig. 2.

Sandblasting and painting tools are embarked on the RCDPR mobile platform. The CoM of the platform follows the profile of the structure tubes and the tools perform the required operations. The paths to be followed, $\mathscr{P}_{1}, \mathscr{P}_{2}$ and $\mathscr{P}_{3}$, are represented in Fig. 2. They are located at a distance of $2 \mathrm{~m}$ from the structure tubes. No path has been assigned to the lower external side of the structure, since it is sandblasted and painted from the ground.

In order to avoid collisions between the cables and the structure, the reconfigurability of the robot anchor point positions is necessary. Each external side of the structure should be painted by one and only one robot configuration. Three configu- 
Fig. 2 Definition of the desired paths, $\mathscr{P}_{1}, \mathscr{P}_{2}$ and $\mathscr{P}_{3}$ of the platform CoM.

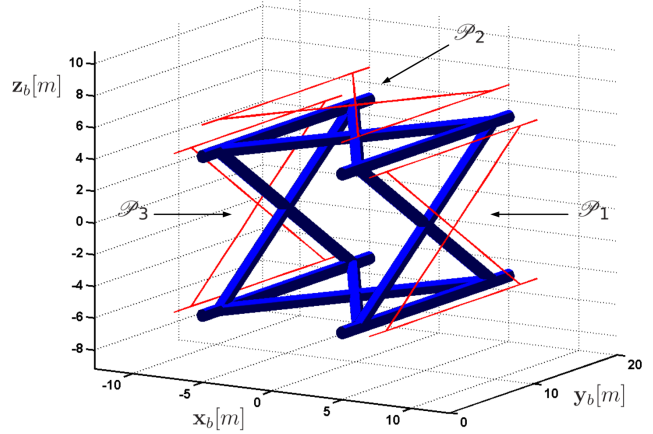

rations are necessary to work at the exterior of the structure: configuration $\mathscr{C}_{i}$ being associated to path $\mathscr{P}_{i}, i=1,2$ and 3 . This requirement is demanded in order not to interrupt the painting and sandblasting operations during their execution. Passing from a configuration to another, one or more cables are disconnected from their anchor points and connected to other anchor points located elsewhere. For each configuration, the locations of the cable exit points are defined as variables of the design problem. In the present work, the dimensions of the platform as well as the position of the cable connection points on the platform are fixed. They will be both detailed in Sec. 4.

A suspended and a fully constrained 8-cable CDPR architecture are considered. The suspended architecture is inspired by the CoGiRo CDPR prototype [13]. For the fully constrained configuration, note that 8 cables is the smallest possible even number of cables that can be used for the platform to be fully constrained by the cables. In the suspended architecture, the static equilibrium of the mobile platform is obtained thanks to the gravity force that plays the role of an additional cable pulling the mobile platform downward.

The RCDPR should be as cheap and simple as possible. For this reason, the minimization of the total number of cable anchor points is required. Consequently, the number of anchor point locations, shared by two or more configurations, should be maximized. The size of the robot is minimized as well, in order to reduce the dimensions of the sandblasting and painting workshop.

Since the sandblasting and painting operations are performed at low speed, the motion of the CDPR platform can be considered to be quasi-static. Hence, only the static equilibrium of the robot mobile platform will be considered. Collisions between the cables as well as collisions between the cables and the structure tubes should be avoided. Besides, the platform positioning precision is constrained as detailed in Sec. 4. Here, the cable mass is not considered. 


\section{RCDPR Kinetostatic Modeling}

A RCDPR is mainly composed of a mobile platform connected to the base through a set of cables, as illustrated in Fig. 3. The connection points of the $i$-th cable on the platform are denoted as $B_{i, c}$, where $c$ represents the configuration number. The position of each point $B_{i, c}$ is expressed by the vector $\mathbf{b}_{i, c}^{p}$ with respect to a local reference frame $\mathscr{F}_{p}$, attached to the platform and of origin $O_{p}$ and axes $\mathbf{x}_{p}, \mathbf{y}_{p}$ and $\mathbf{z}_{p} . O_{p}$ is the platform Center of Mass $(\mathrm{CoM})$. For the $c$-th configuration, the anchor point of the $i$-th cable is denoted by $A_{i, c}, i=1, \ldots, 8$. The Cartesian coordinates of each point $A_{i, c}$, with respect to $\mathscr{F}_{b}$, are given by the vector $\mathbf{a}_{i, c}^{b}$.

The pose of the platform, with respect to $\mathscr{F}_{b}$, is defined by the vector $\mathbf{p}=[\mathbf{t}, \Phi]^{\mathrm{T}}$. The vector $\mathbf{t}$ represents the Cartesian coordinates of the platform CoM. The platform orientation is defined by the vector $\Phi$, through the Euler angles $\phi, \theta$ and $\psi$ corresponding to rotations around $\mathbf{z}_{b}, \mathbf{x}_{b}$ and $\mathbf{y}_{b}$, respectively.

For a given configuration $c$, the vector directed along the cable from $B_{i, c}$ to $A_{i, c}$, expressed in $\mathscr{F}_{b}$, is defined as follows:

$$
\mathbf{l}_{i, c}^{b}=\mathbf{a}_{i, c}^{b}-\mathbf{t}-\mathbf{R} \mathbf{b}_{i, c}^{p} \quad i=1, \ldots, 8
$$

where $\mathbf{R}$ is the rotation matrix defining the platform orientation:

$$
\mathbf{R}=\mathbf{R}_{z}(\phi) \mathbf{R}_{x}(\theta) \mathbf{R}_{y}(\psi)=\left[\begin{array}{ccc}
c \phi c \psi-s \phi s \theta s \psi & -s \phi c \theta & c \phi s \psi+s \phi s \theta c \psi \\
s \phi c \psi+c \phi s \theta s \psi & c \phi c \theta & s \phi s \psi-c \phi s \theta c \psi \\
-c \theta s \psi & s \theta & c \theta c \psi
\end{array}\right]
$$

The unit vector $\mathbf{d}_{i, c}$ associated to each vector $\mathbf{l}_{i, c}$ is given by:

$$
\mathbf{d}_{i, c}^{b}=\frac{\mathbf{l}_{i, c}^{b}}{\left\|\mathbf{l}_{i, c^{b}}\right\|_{2}}, \quad i=1, \ldots, 8
$$

The $i$-th cable exerts on the platform a wrench $\mathbf{w}_{i}$. This wrench is produced by a positive cable tension $\tau_{i}$. All the cable tensions are collected in the vector $\tau=$ $\left[\tau_{1}, \ldots, \tau_{8}\right]^{\mathrm{T}}$. The static equilibrium of the platform is described by the following equation:

$$
\mathbf{W} \tau+\mathbf{w}_{e}=0
$$

where $\mathbf{W}$ is the wrench matrix, defined as follows:

$$
\mathbf{W}=\left[\begin{array}{cccc}
\mathbf{d}_{1, c}^{b} & \mathbf{d}_{2, c}^{b} & \cdots & \mathbf{d}_{8, c}^{b} \\
\mathbf{R b}_{1, c}^{p} \times \mathbf{d}_{1, c}^{b} & \mathbf{R} \mathbf{b}_{2, c}^{p} \times \mathbf{d}_{2, c}^{b} & \cdots & \mathbf{R} \mathbf{b}_{m, c}^{p} \times \mathbf{d}_{8, c}^{b}
\end{array}\right]
$$

The vector $\mathbf{w}_{e}$ is the external wrench. It describes the wrench transmitted by the sandblasting or painting tools to the RCDPR platform and the weight of the platform and of the embarked tools. The weight of the platform and the embarked tools is considered to be equal to $60 \mathrm{Kg}$. 
Fig. 3 Geometric model of a generic RCDPR. In this example, the anchor points are connected to a four-beam structure. Two configurations are illustrated. The anchor point $A_{1,1}$, belonging to the first configuration, is exchanged for the position $A_{1,2}$, belonging to the second configuration.

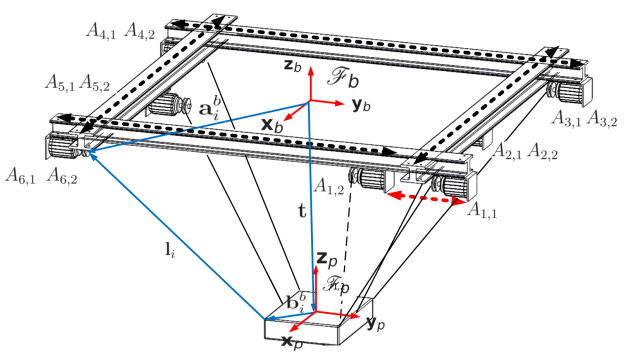

$$
\mathbf{w}_{e}=[\mathbf{f}, \mathbf{m}]^{\mathrm{T}}=\left[f_{x}, f_{y}, f_{z}, m_{x}, m_{y}, m_{z}\right]^{\mathrm{T}}
$$

The forces transmitted along the axes $\mathbf{x}_{b}, \mathbf{y}_{b}$ and $\mathbf{z}_{b}$ are represented by the components $f_{x}, f_{y}$ and $f_{z}$, respectively. The maximum intensity of these components are considered to be equal to $50 \mathrm{~N}$. The maximum values of the transmitted torques $m_{x}$, $m_{y}$ and $m_{z}$, are equal to $7.5 \mathrm{Nm}$.

$$
\begin{gathered}
-50 \mathrm{~N}=f_{\text {min }} \leq f_{x}, f_{y}, f_{z} \leq f_{\text {max }}=50 \mathrm{~N} \\
-7.5 \mathrm{Nm}=m_{\text {min }} \leq m_{x}, m_{y}, m_{z} \leq m_{\text {max }}=7.5 \mathrm{Nm}
\end{gathered}
$$

Cable tensions can be computed from Eq. (4):

$$
\tau=\tau_{n}+\tau_{0}=\mathbf{W}^{\dagger} \mathbf{w}_{e}+\lambda \mathbf{N} \quad \tau \geq \mathbf{0}
$$

$\mathbf{W}^{\dagger}$ is the Moore-Penrose generalized inverse of $\mathbf{W}, \lambda \in \mathbb{R}^{2}$ and $\mathbf{N}$ is the null space of $\mathbf{W}[15]$.

The elastostatic model defines the relationship between the infinitesimal change $\delta \mathbf{p}$ in the platform pose and the corresponding infinitesimal change of the external wrench $\delta \mathbf{w}_{e}$ :

$$
\delta \mathbf{w}_{e}=\mathbf{K} \delta \mathbf{p}
$$

The expression of the stiffness matrix $\mathbf{K}$ is [1]

$$
\begin{aligned}
\mathbf{K}= & \sum_{i=1}^{m} k_{i}\left[\begin{array}{cc}
\mathbf{d}_{i, c} \mathbf{d}_{i, c}^{\mathrm{T}} & \mathbf{d}_{i, c} \mathbf{d}_{i, c}^{\mathrm{T}} \hat{\mathbf{b}}_{i, c}^{b}{ }^{\mathrm{T}} \\
\hat{\mathbf{b}}_{i, c}^{b} \mathbf{d}_{i, c} \mathbf{d}_{i, c}^{\mathrm{T}} & \hat{\mathbf{b}}_{i, c}^{b} \mathbf{d}_{i, c} \mathbf{d}_{i, c}^{\mathrm{T}} \hat{\mathbf{b}}_{i, c}^{b} \mathrm{~T}
\end{array}\right]+ \\
& -\sum_{i=1}^{m} \frac{\tau_{i}}{\left\|\mathbf{l}_{\mathbf{i}}\right\|}\left[\begin{array}{cc}
-\mathbf{I}_{3,3}+\mathbf{d}_{i, c} \mathbf{d}_{i, c}^{\mathrm{T}} & \left(-\mathbf{I}_{3,3}+\mathbf{d}_{i, c} \mathbf{d}_{i, c}^{\mathrm{T}}\right) \hat{\mathbf{b}}_{i, c}^{b} \hat{\mathbf{b}}_{i, c}^{b}\left(-\mathbf{I}_{3,3}+\mathbf{d}_{i, c} \mathbf{d}_{i, c}^{\mathrm{T}}\right)
\end{array}\right]\left(\begin{array}{l}
\left.\left.\hat{\mathbf{b}}_{i, c}^{b}\left(-\mathbf{I}_{3,3}+\mathbf{d}_{i, c} \mathbf{d}_{i}^{\mathrm{T}}\right)+\hat{\mathbf{b}}_{i, c}^{b}\right)^{\mathrm{T}}\right) \hat{\mathbf{b}}_{i, c}^{b}
\end{array}\right]
\end{aligned}
$$

where $k_{i}$ denotes the $i$-th cable longitudinal stiffness, $\mathbf{I}_{3 \times 3}$ denotes the $3 \times 3$ identity matrix, $\hat{\mathbf{b}}_{i, c}^{b}$ and $\hat{\mathbf{d}}_{i, c}$ represent the cross product matrices of vectors $\mathbf{b}_{i, c}^{b}$ and $\mathbf{d}_{i, c}$, respectively. For a generalized 3-dimensional vector $\mathbf{r}=\left[r_{x}, r_{y}, r_{z}\right]^{\mathrm{T}}$, the cross product matrix is defined as follows: 


$$
\hat{\mathbf{r}}=\left[\begin{array}{ccc}
0 & -r_{z} & r_{y} \\
r_{z} & 0 & -r_{x} \\
-r_{y} & r_{x} & 0
\end{array}\right]
$$

\section{Design Procedure}

The design problem aims at identifying the locations of points $A_{i, c}$ for the configurations $\mathscr{C}_{1}, \mathscr{C}_{2}$ and $\mathscr{C}_{3}$. The number of cables, $m$, the cable properties and the dimensions of the platform are given in Sec. 4.1. Those parameters are the same for the three robot configurations.

At first, in order to identify the set of feasible locations for the anchor points $A_{i, c}$, the three robot configurations are parameterized and analysed separately in Secs. 4.2, 4.3 and 4.4. A set of anchor points will be considered feasible if the design constraints are satisfied along the whole path to be followed by the CoM of the platform. The design constraints are listed in Sec. 4.5. Note that each path $\mathscr{P}_{i}, i=1, \ldots, 3$ is discretized into 38 points $\mathrm{P}_{j, i}, j=1, \ldots, 38 \quad i=1, \ldots, 3$

Once the set of feasible solutions have been obtained for each configuration, i.e., for each path, a list of robots with a minimum number of anchor points, $n_{c}$, is extracted from the list of feasible robots. Finally, the most compact robots from the list of robots with a minimum number of anchor points are optimal solutions.

\subsection{Design parameters}

The RCDPR developed in this paper is composed of steel cables. The Young Modulus, $E$, is equal to $100 \mathrm{GPa}$. Their diameter, $\phi_{c}$ is equal to $4 \mathrm{~mm}$, the stiffness coefficient, $k_{i}$, is equal to $252 \mathrm{KN} / \mathrm{m}$. The maximum allowed tension in the cables, $\tau_{\max }$, is equal to $34950 \mathrm{~N}$ :

$$
0<\tau_{i} \leq \tau_{\max }, \quad \forall i=1, \ldots, 8
$$

$l_{p}, w_{p}$ and $h_{p}$ denote the length, width and height of the platform, respectively: $l_{p}=$ $30 \mathrm{~cm}, w_{p}=30 \mathrm{~cm}$ and $h_{p}=60 \mathrm{~cm}$. The design (constant) parameter vector $\mathbf{q}$ is expressed as:

$$
\mathbf{q}=\left[m, \phi_{c}, k_{i}, \tau_{\max }, l_{p}, w_{p}, h_{p}\right]^{\mathrm{T}}
$$

\subsection{Configuration $\mathscr{C}_{1}$}

A fully constrained architecture has been assigned to the configuration $\mathscr{C}_{1}$. As shown in [6], this type of robot architecture can assure the robot static equilibrium while minimizing its size. The anchor points $A_{i, 1}$ have been arranged in a parallelepiped layout. The edges of the parallelepiped are aligned with the axes of frame $\mathscr{F}_{b}$. This 


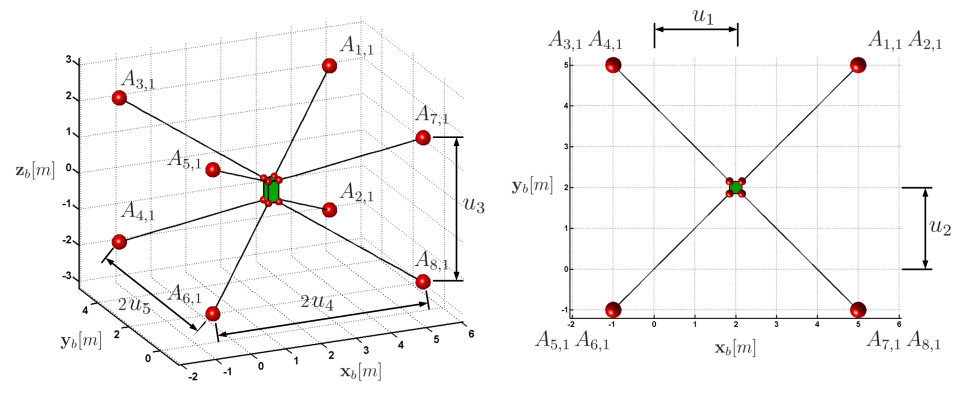

Fig. 4 Design variables parametrizing the configuration $\mathscr{C}_{1}$.

layout can be fully described by means of five variables: $u_{1}, u_{2}$ and $u_{3}$ define the Cartesian coordinates of the parallelepiped centre; $u_{4}$ and $u_{5}$ represent the halflengths of the parallelepiped along the axes $\mathbf{x}_{b}$ and $\mathbf{y}_{b}$, respectively. Therefore, the Cartesian coordinates of the anchor points $A_{i, 1}$ are expressed as follows:

$$
\begin{array}{ll}
\mathbf{a}_{1,1}^{b}=\left[u_{1}+u_{4}, u_{2}+u_{5},-u_{3}\right]^{\mathrm{T}} & \mathbf{a}_{2,1}^{b}=\left[u_{1}+u_{4}, u_{2}+u_{5}, u_{3}\right]^{\mathrm{T}} \\
\mathbf{a}_{3,1}^{b}=\left[u_{1}-u_{4}, u_{2}+u_{5},-u_{3}\right]^{\mathrm{T}} & \mathbf{a}_{4,1}^{b}=\left[u_{1}-u_{4}, u_{2}+u_{5}, u_{3}\right]^{\mathrm{T}} \\
\mathbf{a}_{5,1}^{b}=\left[u_{1}-u_{4}, u_{2}-u_{5},-u_{3}\right]^{\mathrm{T}} & \mathbf{a}_{6,1}^{b}=\left[u_{1}-u_{4}, u_{2}-u_{5}, u_{3}\right]^{\mathrm{T}} \\
\mathbf{a}_{7,1}^{b}=\left[u_{1}+u_{4}, u_{2}-u_{5},-u_{3}\right]^{\mathrm{T}} & \mathbf{a}_{8,1}^{b}=\left[u_{1}+u_{4}, u_{2}-u_{5}, u_{3}\right]^{\mathrm{T}}
\end{array}
$$

The layout of the first robot configuration is described in Fig. 4. The design variables of the design problem at hand are collected into the vector $\mathbf{x}_{1}$ :

$$
\mathbf{x}_{1}=\left[u_{1}, u_{2}, u_{3}, u_{4}, u_{5}\right]^{\mathrm{T}}
$$

The Cartesian coordinates of the connection points $B_{i, 1}$ of the cables to the platform are expressed as:

$$
\begin{array}{rlrl}
\mathbf{b}_{1,1}^{b} & =\frac{1}{2}\left[l_{p}, w_{p}, h_{p}\right]^{\mathrm{T}}, & \mathbf{b}_{2,1}^{b} & =\frac{1}{2}\left[l_{p}, w_{p},-h_{p}\right]^{\mathrm{T}} \\
\mathbf{b}_{3,1}^{b}=\frac{1}{2}\left[-l_{p}, w_{p}, h_{p}\right]^{\mathrm{T}}, & \mathbf{b}_{4,1}^{b}=\frac{1}{2}\left[-l_{p}, w_{p},-h_{p}\right]^{\mathrm{T}} \\
\mathbf{b}_{5,1}^{b}=\frac{1}{2}\left[-l_{p},-w_{p}, h_{p}\right]^{\mathrm{T}}, & \mathbf{b}_{6,1}^{b}=\frac{1}{2}\left[-l_{p},-w_{p},-h_{p}\right]^{\mathrm{T}} \\
\mathbf{b}_{7,1}^{b}=\frac{1}{2}\left[l_{p},-w_{p}, h_{p}\right]^{\mathrm{T}}, & \mathbf{b}_{8,1}^{b}=\frac{1}{2}\left[l_{p},-w_{p},-h_{p}\right]^{\mathrm{T}}
\end{array}
$$

A discretized set of design variables have been considered. The lower and upper bounds as well as the number of values for each variable are given in Tab. 1 . 18225 robot configurations have been generated with those values. It turns out that 4576 configurations satisfy the design constraints expressed in Sec. 4.5 along the 38 discretized points of path $\mathscr{P}_{1}$. 


\subsection{Configuration $\mathscr{C}_{2}$}

A suspended architecture has been attributed to the configuration $\mathscr{C}_{2}$ in order to avoid any possible collision with the tubular structure. The selected architecture is based on CoGiRo, a suspended CDPR designed and built in the framework of the ANR CoGiRo project [13], [11]. An advantage of this architecture is the possibility to balance efficiently the external wrench throughout a very large part of the robot footprint. The anchor points $A_{i, 2}$ have been arranged in a parallelepiped layout. The Cartesian coordinates $\mathbf{a}_{i, c}$ are defined as follows:

$$
\begin{aligned}
\mathbf{a}_{1,2}^{b} & =\mathbf{a}_{2,2}^{b}=\left[v_{1}-v_{4}, v_{2}-v_{5}, v_{3}\right]^{\mathrm{T}} \\
\mathbf{a}_{3,2}^{b} & =\mathbf{a}_{4,2}^{b}=\left[v_{1}-v_{4}, v_{2}+v_{5}, v_{3}\right]^{\mathrm{T}} \\
\mathbf{a}_{5,2}^{b} & =\mathbf{a}_{6,2}^{b}=\left[v_{1}+v_{4}, v_{2}+v_{5}, v_{3}\right]^{\mathrm{T}} \\
\mathbf{a}_{7,2}^{b} & =\mathbf{a}_{7,2}^{b}=\left[v_{1}+v_{4}, v_{2}-v_{5}, v_{3}\right]^{\mathrm{T}}
\end{aligned}
$$

Variables $v_{1}=1, \ldots, 5$ assume the same geometric roles assigned to variables $u_{1}=1, \ldots, 5$. The layout of this configuration is illustrated in Fig. 5. The design variables of configuration $\mathscr{C}_{2}$ are collected into the vector $\mathbf{x}_{2}$ :

$$
\mathbf{x}_{2}=\left[v_{1}, v_{2}, v_{3}, v_{4}, v_{5}\right]^{\mathrm{T}}
$$

Note that this architecture is composed of couples of anchor points theoretically connected to the same locations: $\left\{A_{1,2}, A_{2,2}\right\},\left\{A_{3,2}, A_{4,2}\right\},\left\{A_{5,2}, A_{6,2}\right\}$ and $\left\{A_{7,2}, A_{8,2}\right\}$. From a technical point of view, in order to avoid any cable interference, the coupled anchor points should be separated by a distance at least greater than cable diameter. For the design problem at hand, this distance has been fixed to $5 \mathrm{~mm}$.

The Cartesian coordinates of points $B_{i, 2}$ are defined as:

$$
\begin{aligned}
\mathbf{b}_{1,2}^{b} & =\frac{1}{2}\left[l_{p},-w_{p}, h_{p}\right]^{\mathrm{T}}, & \mathbf{b}_{2,2}^{b} & =\frac{1}{2}\left[-l_{p}, w_{p},-h_{p}\right]^{\mathrm{T}} \\
\mathbf{b}_{3,2}^{b} & =\frac{1}{2}\left[-l_{p},-w_{p}, h_{p}\right]^{\mathrm{T}}, & \mathbf{b}_{4,2}^{b} & =\frac{1}{2}\left[l_{p}, w_{p},-h_{p}\right]^{\mathrm{T}} \\
\mathbf{b}_{5,2}^{b} & =\frac{1}{2}\left[-l_{p}, w_{p}, h_{p}\right]^{\mathrm{T}}, & \mathbf{b}_{6,2}^{b} & =\frac{1}{2}\left[l_{p},-w_{p},-h_{p}\right]^{\mathrm{T}} \\
\mathbf{b}_{7,2}^{b} & =\frac{1}{2}\left[l_{p}, w_{p}, h_{p}\right]^{\mathrm{T}}, & \mathbf{b}_{8,2}^{b} & =\frac{1}{2}\left[-l_{p},-w_{p},-h_{p}\right]^{\mathrm{T}}
\end{aligned}
$$

Table 1 describes the lower and upper bounds as well as the number of values considered for the configuration $\mathscr{C}_{2}$. Combining these values, 22275 configurations have been generated. Amongst these configurations, only 5579 configurations are valid. 

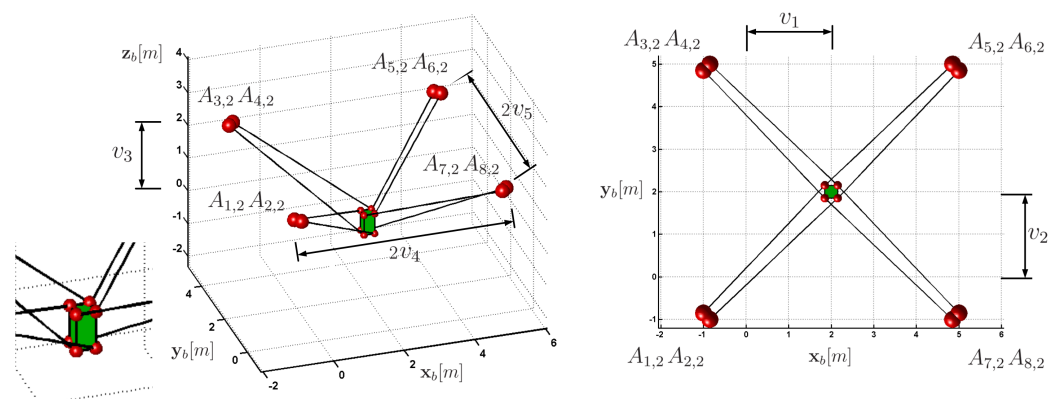

Fig. 5 Design variables parametrizing the configuration $\mathscr{C}_{2}$.

\subsection{Configuration $\mathscr{C}_{3}$}

The configuration $\mathscr{C}_{3}$ follows the path $\mathscr{P}_{3}$. This path is symmetric to the path $\mathscr{P}_{1}$ with respect to the plane $\mathbf{y}_{b} O \mathbf{z}_{b}, O, \mathbf{y}_{b}$ and $\mathbf{z}_{b}$ being the origin, the $y$-axis and the $z$ axis of the base frame. Considering the symmetry of the tubular structure, the robot architecture used for the configuration $\mathscr{C}_{1}$ has been assigned to the configuration $\mathscr{C}_{3}$. The discretized set of design variables chosen for the configuration $\mathscr{C}_{3}$ is described in Tab. 1. The design variables for the configuration $\mathscr{C}_{3}$ are collected into the vector $\mathbf{x}_{3}$ :

$$
\mathbf{x}_{3}=\left[w_{1}, w_{2}, w_{3}, w_{4}, w_{5}\right]^{\mathrm{T}}
$$

where the variables $w_{i}$ amount to variables $u_{i}$ described in Sec. 4.2. Therefore, the Cartesian coordinates of the anchor points $A_{i, 3}$ are expressed as follows:

$$
\begin{aligned}
\mathbf{a}_{1,3}^{b}=\left[w_{1}+w_{4}, w_{2}+w_{5},-w_{3}\right]^{\mathrm{T}} & \mathbf{a}_{2,3}^{b}=\left[w_{1}+w_{4}, w_{2}+w_{5}, w_{3}\right]^{\mathrm{T}} \\
\mathbf{a}_{3,3}^{b}=\left[w_{1}-w_{4}, w_{2}+w_{5},-w_{3}\right]^{\mathrm{T}} & \mathbf{a}_{4,3}^{b}=\left[w_{1}-w_{4}, w_{2}+w_{5}, w_{3}\right]^{\mathrm{T}} \\
\mathbf{a}_{5,3}^{b}=\left[w_{1}-w_{4}, w_{2}-w_{5},-w_{3}\right]^{\mathrm{T}} & \mathbf{a}_{6,3}^{b}=\left[w_{1}-w_{4}, w_{2}-w_{5}, w_{3}\right]^{\mathrm{T}} \\
\mathbf{a}_{7,3}^{b}=\left[w_{1}+w_{4}, w_{2}-w_{5},-w_{3}\right]^{\mathrm{T}} & \mathbf{a}_{8,3}^{b}=\left[w_{1}+w_{4}, w_{2}-w_{5}, w_{3}\right]^{\mathrm{T}}
\end{aligned}
$$

\subsection{Constraints}

The static equilibrium of the robot should be assured during the entire task execution, balancing any possible external wrench $\mathbf{w}_{e}$, according to the limits specified in Eq. (7) and Eq. (8). This condition is verified for all the points $\mathscr{P}_{i}, i=1, \ldots, 3$. The set of required external wrenches consists of a hyperrectangle, defined as $[\mathbf{w}]_{r}$. In order the robot to be in a static equilibrium in a given posture, the hyperrectangle of the required external wrench should be included inside the zonotope of the admissible wrench, $[\mathbf{w}]_{a}$, as follows: 
Table 1 Design variables associated with configurations $\mathscr{C}_{1}, \mathscr{C}_{2}$ and $\mathscr{C}_{3}$.

\begin{tabular}{|c|c|c|c|c|}
\hline & Variables & Lower Bounds & Upper Bounds & Number of values \\
\hline \multirow{5}{*}{$\mathscr{C}_{1}$} & $u_{1}$ & 5.5 & 7.5 & 9 \\
\hline & $u_{2}$ & 8.0 & 12.0 & 9 \\
\hline & $u_{3}$ & 6 & 10 & 5 \\
\hline & $u_{4}$ & 0.5 & 2.5 & 9 \\
\hline & $u_{5}$ & 10 & 14 & 5 \\
\hline \multirow{5}{*}{$\mathscr{C}_{2}$} & $v_{1}$ & -1 & 1 & 9 \\
\hline & $v_{2}$ & 8.0 & 12.0 & 5 \\
\hline & $v_{3}$ & 7 & 11 & 9 \\
\hline & $v_{4}$ & 5 & 7.5 & 11 \\
\hline & $v_{5}$ & 10 & 14 & 5 \\
\hline \multirow{5}{*}{$\mathscr{C}_{3}$} & $u_{1}$ & -7.5 & -5.5 & 9 \\
\hline & $u_{2}$ & 8.0 & 12.0 & 9 \\
\hline & $u_{3}$ & 6 & 10 & 5 \\
\hline & $u_{4}$ & 0.5 & 2.5 & 9 \\
\hline & $u_{5}$ & 10 & 14 & 5 \\
\hline
\end{tabular}

$$
\forall \mathbf{w}_{e} \in[\mathbf{w}]_{r}, \exists \tau \in[\tau] \text { such that }\left\{\begin{array}{l}
{[\mathbf{w}]_{r} \in[\mathbf{w}]_{a}} \\
\mathbf{W} \tau+\mathbf{w}_{e}=0
\end{array}\right.
$$

$[\mathbf{w}]_{a}$ represents the possible external wrenches that the platform can balance. $[\mathbf{w}]_{a}$ depends on the robot geometry and the cable tension limits, defined by Eq. (13). Any mobile platform pose respecting the previous condition is said to be wrench feasible. The set of wrench feasible poses represents the Wrench Feasible Workspace (WFW) of the given robot.

In [8], Gouttefarde et al. improved the wrench feasibility verification procedure defined by Bouchard et al. in [4]. Both the procedures lead to a set of inequalities:

$$
\mathbf{C w} \leq \mathbf{d}, \quad \forall \mathbf{w} \in[\mathbf{w}]_{r}
$$

Cable interferences have to be avoided. The interference between the $i$-th cable and the $j$-th cable is verified analysing the distance $d_{i, j}^{c c}$ between them. The cables have been modelled as linear segments, neglecting the mass and elastic effects on the sagging phenomenon. A fast computation of $d_{i, j}^{c c}$ is realised through Lumelsy's approach [14]. For the constraint to be satisfied, distance $d_{i, j}^{c c}$ should be greater than the diameter of the cables, $\phi_{c}$, i.e.,

$$
d_{i, j}^{c c} \geq \phi_{c} \quad \forall i, j=1, \ldots, m, \quad i \neq j
$$

The same approach is used to detect collisions between the cables and the tubular structure. The collision between the $i$-th cable and the $k$-th structure tube does not occur when their distance $d_{i, k}^{c c}$ is greater than the sum of the cable and tube radii:

$$
d_{i, k}^{c s} \geq \frac{\left(\phi_{c}+\phi_{s}\right)}{2} \quad \forall i=1, \ldots, m, \quad \forall k=1, \ldots, 12
$$


The RCDPR should respect a prescribed pose accuracy along all the paths. Inside the limits defined by the required wrench set, the maximum mobile platform linear displacements $\delta t_{x}, \delta t_{y}$ and $\delta t_{z}$ along the $x_{b}, y_{b}$ and $z_{b}$ axes should be lower than $5 \mathrm{~cm}$ :

$$
-5 \mathrm{~cm} \leq \delta t_{x}, \delta t_{y}, \delta t_{z} \leq 5 \mathrm{~cm}
$$

The rotation displacements of the platform $\delta r_{x}, \delta r_{y}$ and $\delta r_{z}$ about axes $\mathbf{x}_{b}, \mathbf{y}_{b}$ and $\mathbf{z}_{b}$ should not be higher than $0.1 \mathrm{rad}$ :

$$
-0.1 \mathrm{rad} \leq \delta r_{x}, \delta r_{y}, \delta r_{z} \leq 0.1 \mathrm{rad}
$$

The linear and rotational displacements of the mobile platform are computed from Eq. (10).

\subsection{Objective Functions}

In Secs. 4.2, 4.3 and 4.4, the feasible robot configurations associated to paths $\mathscr{P}_{1}$, $\mathscr{P}_{2}$ and $\mathscr{P}_{3}$ have been identified. For each path, a configuration is selected, aiming at minimizing the total number of anchor points required by the RCDPR to complete the task. These optimal solutions have been computed in two phases. At first, the 4576 feasible robot configurations for path $\mathscr{P}_{1}$ are compared with the 5579 feasible robot configurations for path $\mathscr{P}_{2}$. The sets of robot configurations that minimize the number, $n_{c}$, of required anchor points along the three paths are selected.

Then, the problem aims to minimize the size of the robot, defined as the convex hull of the robot anchor points. The Cartesian coordinates of anchor point $A_{i, c}$ are defined as $\mathbf{a}_{i, c}=\left[a_{i, c}^{x}, a_{i, c}^{y}, a_{i, c}^{z}\right]^{\mathrm{T}}$. The variables $\underline{s}_{x}, \underline{s}_{y}$ and $\underline{s}_{z}$ denote the smallest Cartesian coordinates of the robot anchor points along the axes $\mathbf{x}_{b}, \mathbf{y}_{b}$ and $\mathbf{z}_{b}$, respectively:

$$
\begin{aligned}
& \underline{s}_{x}=\min a_{i, c}^{x}, \forall i=1, \ldots, 8, c=1, \ldots, 3 \\
& \underline{s}_{y}=\min a_{i, c}^{y}, \forall i=1, \ldots, 8, c=1, \ldots, 3 \\
& \underline{s}_{z}=\min a_{i, c}^{z}, \forall i=1, \ldots, 8, c=1, \ldots, 3
\end{aligned}
$$

The upper bounds on the Cartesian coordinates of the RCDPR anchor points, along the axes $\mathbf{x}_{b}, \mathbf{y}_{b}, \mathbf{z}_{b}$, are denoted by $\bar{s}_{x}, \bar{s}_{y}$ and $\bar{s}_{z}$, respectively.

$$
\begin{aligned}
& \bar{s}_{x}=\max a_{i, c}^{x}, \forall i=1, \ldots, 8, c=1, \ldots, 3 \\
& \bar{s}_{y}=\max a_{i, c}^{y}, \forall i=1, \ldots, 8, c=1, \ldots, 3 \\
& \bar{s}_{z}=\max a_{i, c}^{z}, \forall i=1, \ldots, 8, c=1, \ldots, 3
\end{aligned}
$$

Hence, the objective function related to the size of the robot is expressed as follows:

$$
\mathscr{N}_{2}=\left(\bar{s}_{x}-\underline{s}_{x}\right)\left(\bar{s}_{y}-\underline{s}_{y}\right)\left(\bar{s}_{z}-\underline{s}_{z}\right)
$$




\subsection{Design Problem Formulation}

The design problem of the CDPR is formulated as follows:

$$
\begin{aligned}
& \operatorname{minimize}\left\{\begin{array}{l}
\mathscr{V}_{1}=n_{c} \\
\mathscr{V}_{2}=\left(\bar{s}_{x}-\underline{s}_{x}\right)\left(\bar{s}_{y}-\underline{s}_{y}\right)\left(\bar{s}_{z}-\underline{s}_{z}\right)
\end{array}\right. \\
& \text { over } \mathbf{x}_{1}, \mathbf{x}_{2}, \mathbf{x}_{3} \\
& \begin{array}{r}
\forall \mathrm{P}_{m, n}, m=1, \ldots, 38 \\
n=1, \ldots, 3
\end{array}\left\{\begin{array}{l}
\mathbf{C w} \leq \mathbf{d}, \quad \forall \mathbf{w} \in[\mathbf{w}]_{r} \\
d_{i, j}^{c c} \geq \phi_{c} \quad \forall i, j=1, \ldots, 8, \quad i \neq j \\
d_{i, k}^{c s} \geq \frac{\left(\phi_{c}+\phi_{s}\right)}{2} \quad \forall i=1, \ldots, 8 \\
\forall k=1, \ldots, 12 \\
-5 \mathrm{~cm} \leq \delta t_{x}, \delta t_{y}, \delta t_{z} \leq 5 \mathrm{~cm} \\
-0.1 \mathrm{rad} \leq \delta r_{x}, \delta r_{y}, \delta r_{z} \leq 0.1 \mathrm{rad}
\end{array}\right.
\end{aligned}
$$

\section{Results}

Following the procedure described in Sec. 4, a set of feasible configurations have been identified for $\mathscr{C}_{l}, l=1, \ldots, 3.16516$ triplets of configurations minimize the total number of anchor points.

A generic CDPR composed of 8 cables requires 8 anchor points $A_{i}=1, \ldots, 8$ on the base. It is the case for the fully-constrained configuration $\mathscr{C}_{1}$ described in Sec. 4.2. The suspended CDPR proposed in Sec. 4.3 presents 4 coincident couples of anchor points. Hence, the maximum total number of anchor points of the RCDPR is equal to 20 . The best results provide a reduction of 4 points. Regarding the configurations $\mathscr{C}_{1}$ and $\mathscr{C}_{2}$, points $A_{5,2}$ and $A_{7,2}$ can be coincident with points $A_{3,1}$ and $A_{5,1}$, respectively. Alternatively, points $A_{5,2}$ and $A_{7,2}$ can be coincident with points $A_{1,1}$ and $A_{7,1}$. As far as configurations $\mathscr{C}_{2}$ and $\mathscr{C}_{3}$ are concerned, points $A_{1,2}$ and $A_{3,2}$ can be coincident with points $A_{8,3}$ and $A_{2,3}$, respectively. Likewise, points $A_{1,2}$ and $A_{3,2}$ can be coincident with points $A_{4,3}$ and $A_{6,3}$, respectively.

The total volume of the robot has been computed for the 16516 configurations minimizing the total number of anchor points. 96 RCDPRs amongst the 16516 robot configurations have the smallest size, this minimum size being equal to $5104 \mathrm{~m}^{3}$. An optimal solution is illustrated in Fig. 6. The corresponding optimal design parameters are given in Tab. 2.

Fig. 7 illustrates the minimum degree of constraint satisfaction introduced in [9] and defined thereafter along the paths $\mathscr{P}_{1}, \mathscr{P}_{2}$ and $\mathscr{P}_{3}$, which is discretized into 388 points. 

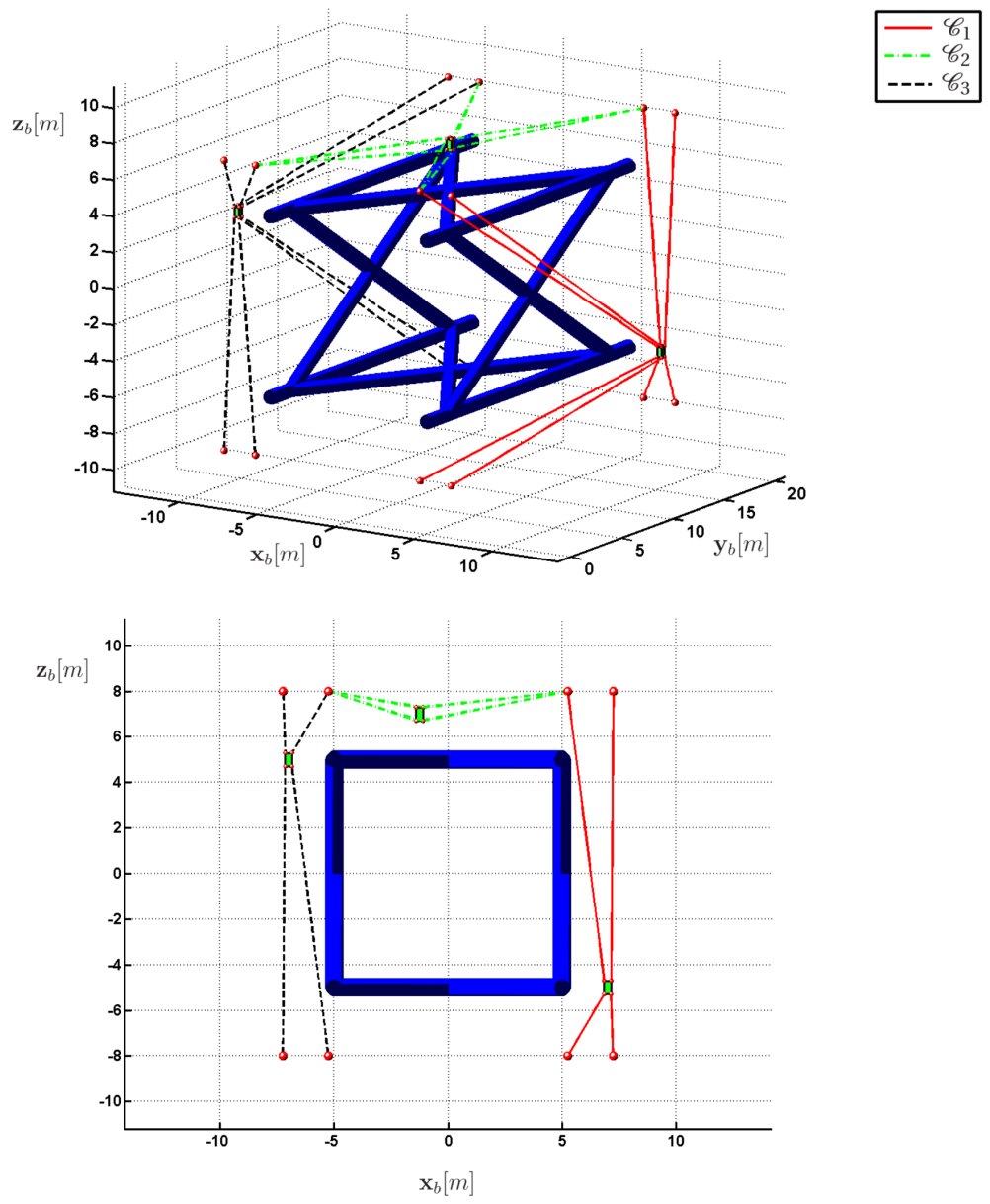

Fig. 6 Optimal Reconfigurable Cable-Driven Parallel Robot.

$$
s=\min _{i=1, \ldots, n}\left(\min _{j=1, \ldots, p} s_{j, i}\right)
$$

It turns out that mobile platform is in a static equilibrium along all the paths because the minimum degree of constraint satisfaction remains negative. The degree of constraint satisfaction $s_{j, i}$ is the signed distance from the $j$-th vertex $\mathbf{w}_{e, j}$ of $[\mathbf{w}]_{r}$ to the $i$-th face of $[\mathbf{w}]_{a} . s_{j, i}$ is negative when a pose is wrench feasible. Configurations $\mathscr{C}_{1}$ and $\mathscr{C}_{3}$ maintain their degree of satisfaction lower than the $400 \mathrm{~N}$. On the contrary, configuration $\mathscr{C}_{2}$ is often close to 0 . The poses where $s$ vanishes require that two cables of the suspended CDPR are slack. 


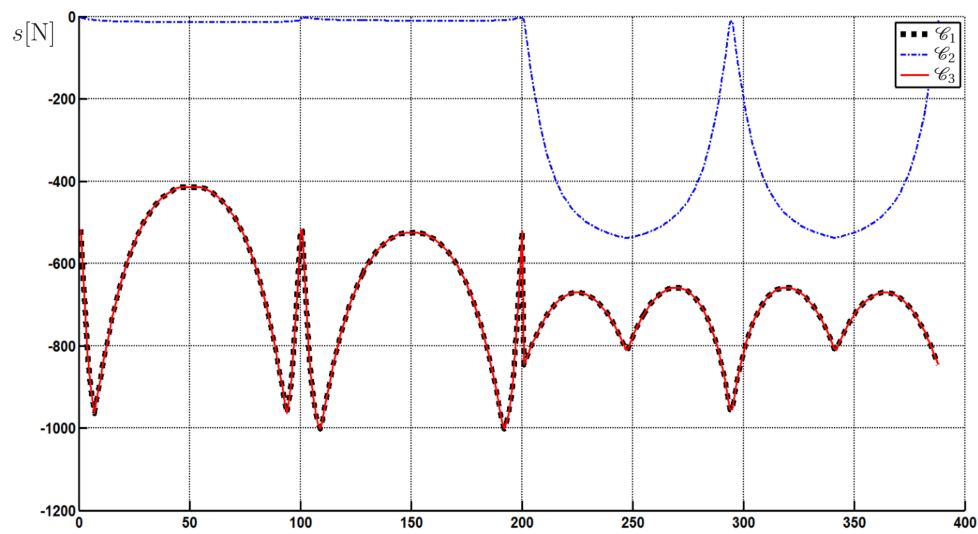

Fig. 7 Minimum degree of constraint satisfaction. The analysis has been performed by discretizing the paths $\mathscr{P}_{1}, \mathscr{P}_{2}$ and $\mathscr{P}_{3}$ into 388 points.

Table 2 Design parameters of the selected optimum RCDPR.

\begin{tabular}{cccccc}
\hline \hline Conf. & var.1 & var.2 & var.3 & var.4 & var.5 \\
\hline $\mathbf{x}_{1}$ & 6.25 & 10.0 & 8.0 & 1.0 & 11.0 \\
$\mathbf{x}_{3}$ & 0 & 10.0 & 8.0 & 5.25 & 11.0 \\
$\mathbf{x}_{3}$ & -6.25 & 10.0 & 8.0 & 1.0 & 11.0 \\
\hline \hline
\end{tabular}

\section{Conclusions}

This paper dealt with the design of a Reconfigurable Cable Driven Parallel Robot (RCDPR) for sandblasting and painting of large structures. A tubular structure of $20 \mathrm{~m}$ long and $10 \mathrm{~m}$ wide was considered. The design problem aimed at determining the optimal locations of the cable anchor points such that the number of anchor points is minimized and the RCDPR is as small as possible. The problem solving led to 96 optimal solutions.

A RCDPR was required in order to complete a given task in a complex environment. Unfortunately, the design strategy introduced in this paper is very time consuming. The whole procedure, performed on a Intel ${ }^{\circledR}$ Core $^{\mathrm{TM}}$ i7-3630QM $2.40 \mathrm{GHz}$, required $19 \mathrm{~h}$ of computation, on Matlab ${ }^{\circledR}$ 2013a. Therefore, the development of more efficient strategies for the design of RCDPRs will be part of our future work. Moreover, the mass of the cables should be taken into account.

Acknowledgements This research work has been supported by the IRT Jules Verne, Bouguenais, France (Project CAROCA, Evaluation des CApacités de la RObotique à CÂbles dans un contexte industriel). 


\section{References}

1. S. Behzadipour and A. Khajepour. Stiffness of cable-based parallel manipulators with application to stability analysis. J. Mechanical Design, 128:303-310, 2006.

2. R. Bostelman, J. Albus, N. Dagalakis, and A. Jacoff. Applications of the NIST RoboCrane. In Proceedings of the 5th International Symposium on Robotics and Manufacturing, Maui, Aug. 1994.

3. R. Bostelman, A. Jacoff, F. Proctor, T. Kramer, and A. Wavering. Cable-based reconfigurable machines for large scale manufacturing. In Proc. of the 2000 Japan-USA Symposium on Flexible Automation, Ann Arbor, MI, July 2000.

4. S. Bouchard, C. M. Gosselin, and B. Moore. On the ability of a cable-driven robot to generate a prescribed set of wrenches. In Proc. of the ASME 2008 Int. Design Eng. Technical Conf. \& Computers and Information in Eng. Conf., pages 1-12, Brooklyn, New York, USA,, Aug. 2008.

5. CableBOT [Online]. Available: http://www.cablebot.eu/en/.

6. L. Gagliardini, S. Caro, M. Gouttefarde, P. Wenger, and A. Girin. Optimal design of cabledriven parallel robots for large industrial structures. In IEEE International Conference on Robotics and Automation (ICRA 2014), Hong Kong, China, May 2014.

7. M. Gouttefarde, D. Daney, and J.-P. Merlet. Interval-analysis-based determination of the wrench-feasible workspace of parallel cable-driven robots. IEEE Transactions on Robotics, 27(1):1-13, 2011.

8. M. Gouttefarde and S. Krut. Characterization of parallel manipulator available wrench set facets. In Advances in Robot Kinematics: Motion in Man and Machine, pages 475-484. Piran, 2010.

9. F. Guay, P. Cardou, A.L. Cruz, and S. Caro. Measuring how well a structure supports varying external wrenches. In The Second Conference on Mechanisms, Transmissions and Applications, Bilbao, Spain, Oct. 2013.

10. C.S. Holland and D.J. Cannon. Cable array robot for material handling, March 2002.

11. CoGiRo [Online]. Available: http://www.lirmm.fr/cogiro/.

12. J.-B Izard, M. Gouttefarde, O. Tempier, and C. Baradat. A reconfigurable robot for cabledriven parallel robotic research and industrial scenario proofing. In Cable-Driven Parallel Robots, volume 12, pages 135-148. 2013.

13. J. Lamaury, M. Gouttefarde, A. Chemori, and P.-E. Herve. Dual-space adaptive control of redundantly actuated cable-driven parallel robots. In IEEE/RSJ International Conference on Intelligent Robots and Systems (IROS), pages 4879-4886, Tokyo, Japan, Nov. 2013.

14. V.J. Lumelsky. On fast computation of distance between line segments. Information Processing Letters, 21, 1985.

15. R.G. Roberts, T. Graham, and T. Lippitt. On the inverse kinematics, statics, and fault tolerance of cable-suspended robots. J. of Robotic Systems, 15(10):581-597, 1998.

16. G. Rosati, D. Zanotto, and S. K. Agrawal. On the design of adaptive cable-driven systems. Journal of Mechanisms and Robotics, 3, May 2011.

17. A. Zhou, C. P. Tang, and V. Krovi. Analysis framework for cooperating mobile cable robots. In IEEE Int. Conf. on Robotics and Automation, pages 3128-3133, RiverCentre, Saint Paul, MN, May 2012. 